TRANSACTIONS OF THE

AMERICAN MATHEMATICAL SOCIETY

Volume 351, Number 1, January 1999, Pages 363-377

S 0002-9947(99)02235-7

\title{
ON SECTIONAL GENUS OF QUASI-POLARIZED 3-FOLDS
}

\author{
YOSHIAKI FUKUMA
}

\begin{abstract}
Let $X$ be a smooth projective variety over $\mathbb{C}$ and $L$ a nef-big (resp. ample) divisor on $X$. Then $(X, L)$ is called a quasi-polarized (resp. polarized) manifold. Then we conjecture that $g(L) \geq q(X)$, where $g(L)$ is the sectional genus of $L$ and $q(X)=\operatorname{dim} H^{1}\left(\mathcal{O}_{X}\right)$ is the irregularity of $X$. In general it is unknown whether this conjecture is true or not, even in the case of $\operatorname{dim} X=2$. For example, this conjecture is true if $\operatorname{dim} X=2$ and $\operatorname{dim} H^{0}(L)>0$. But it is unknown if $\operatorname{dim} X \geq 3$ and $\operatorname{dim} H^{0}(L)>0$. In this paper, we prove $g(L) \geq q(X)$ if $\operatorname{dim} X=3$ and $\operatorname{dim} H^{0}(L) \geq 2$. Furthermore we classify polarized manifolds $(X, L)$ with $\operatorname{dim} X=3, \operatorname{dim} H^{0}(L) \geq 3$, and $g(L)=q(X)$.
\end{abstract}

\section{INTRODUCTION}

Let $(X, L)$ be a quasi-polarized manifold with $\operatorname{dim} X=n$. For this $(X, L)$, the sectional genus $g(L)$ is defined by the following formula:

$$
g(L)=1+\frac{1}{2}\left(K_{X}+(n-1) L\right) L^{n-1} .
$$

Then there is the following conjecture which is interesting and difficult.

Conjecture. Let $(X, L)$ be a quasi-polarized manifold. Then $g(L) \geq q(X)$, where $q(X)=\operatorname{dim} H^{1}\left(\mathcal{O}_{X}\right)$.

For this Conjecture, there are some results (see [Fk1], [Fk2], [Fk3]). But it is unknown whether this Conjecture is true or not, even in the case of $\operatorname{dim} X=2$. If $\operatorname{dim} X=2$, then this Conjecture is true if $h^{0}(L)=\operatorname{dim} H^{0}(L)>0$. This proof is easy (see [Fk1]). But if $\operatorname{dim} X \geq 3$, it is unknown whether this Conjecture is true or not, even in the case $h^{0}(L)>0$. In this paper, we study the case in which $\operatorname{dim} X=3$ and $h^{0}(L) \geq 2$.

In the paper [Fk4], we proved that $g(L) \geq(2 / 3) q(X)+1 / 3$ if $\kappa(X) \geq 0$ and $h^{0}(L) \geq 2$. But we can improve this result.

First we will prove the following theorem.

Theorem 2.1. Let $(X, L)$ be a quasi-polarized 3-fold with $h^{0}(L) \geq 2$. Then $g(L) \geq$ $q(X)$.

The method of the proof of Theorem 2.1 is thought to be the best way to prove the Conjecture if $h^{0}(L) \geq 2$, and we can find out that this Conjecture is related to the minimal model problem (in particular the Flip Conjecture). (See Theorem 2.5.)

Furthermore if $\operatorname{dim} X=3, h^{0}(L) \geq 3$, and $g(L)=q(X)$, then we can classify the type of polarized 3 -folds $(X, L)$ as follows.

Received by the editors February 5, 1997.

1991 Mathematics Subject Classification. Primary 14C20; Secondary 14J99. 
Theorem 2.12. Let $(X, L)$ be a polarized 3-fold with $h^{0}(L) \geq 3$. If $g(L)=q(X)$, then $(X, L)$ is one of the following types:

1. $\Delta(L)=0$;

2. $(X, L)$ is a scroll over a curve.

The case in which $h^{0}(L)=1$ will be studied in a forthcoming paper.

The author would like to express his hearty gratitude to Professor Takao Fujita for giving some useful comments.

\section{Preliminaries}

Definition 1.1. The pair $(X, L)$ is called a quasi-polarized (resp. polarized) manifold if $X$ is a smooth projective variety over $\mathbb{C}$ and $L$ is a nef-big (resp. an ample) line bundle. $(f, X, Y)$ is called a fiber space if $X$ and $Y$ are smooth projective varieties over $\mathbb{C}$ with $\operatorname{dim} X>\operatorname{dim} Y \geq 1$ and $f$ is a surjective morphism $X \rightarrow Y$ with connected fibers. $(f, X, Y, L)$ is called a quasi-polarized (resp. polarized) fiber space if $(f, X, Y)$ is a fiber space and $L$ is a nef-big (resp. an ample) line bundle.

Definition 1.2. (1) Let $\left(X_{1}, L_{1}\right)$ and $\left(X_{2}, L_{2}\right)$ be quasi-polarized manifolds, where $X_{i}$ may have singularities for $i=1,2$. Then $\left(X_{1}, L_{1}\right)$ and $\left(X_{2}, L_{2}\right)$ are said to be birationally equivalent if there is another variety $G$ with birational morphisms $g_{i}: G \rightarrow X_{i}(i=1,2)$ such that $g_{1}^{*} L_{1}=g_{2}^{*} L_{2}$.

(2) Let $\left(f_{1}, X_{1}, Y, L_{1}\right)$ and $\left(f_{2}, X_{2}, Y, L_{2}\right)$ be quasi-polarized fiber spaces, where $X_{i}$ may have singularities for $i=1,2$. Then $\left(f_{1}, X_{1}, Y, L_{1}\right)$ and $\left(f_{2}, X_{2}, Y, L_{2}\right)$ are said to be birationally equivalent if there is another variety $G$ with birational morphisms $g_{i}: G \rightarrow X_{i}(i=1,2)$ such that $g_{1}^{*} L_{1}=g_{2}^{*} L_{2}$ and $f_{1} \circ g_{1}=f_{2} \circ g_{2}$.

Theorem 1.3. Let $(f, X, C, L)$ be a polarized fiber space with $\operatorname{dim} X=n \geq 3$. Then

(1) $K_{X / C}+(n-1) L$ is nef unless $(f, X, C, L)$ is a scroll.

(2) $g(L) \geq g(C)$ holds and if this equality holds, then $(f, X, C, L)$ is a scroll.

Proof. See Theorem 1.1.2, Theorem 1.2.1, and Theorem 1.4.2 in [Fk2].

Theorem 1.4. Let $(X, L)$ be a quasi-polarized 3-fold. Then there exists a quasipolarized variety $\left(X^{\prime}, L^{\prime}\right)$ which is birationally equivalent to $(X, L)$ and satisfies one of the following conditions:

1. $K_{X^{\prime}}+2 L^{\prime}$ is nef for the canonical $\mathbb{Q}$-bundle $K_{X^{\prime}}$,

2. $\Delta\left(L^{\prime}\right)=0$,

3. $\left(X^{\prime}, L^{\prime}\right)$ is a scroll over a curve,

where $X^{\prime}$ is a normal projective variety with only $\mathbb{Q}$-factorial terminal singularities.

Proof. See Theorem 4.2 in [Fj2].

Theorem 1.5. Let $(f, X, C, L)$ be a quasi-polarized fiber space with $\operatorname{dim} X=3$ and $\operatorname{dim} C=1$. Then there exists a quasi-polarized fiber space $\left(f^{\prime}, X^{\prime}, C, L^{\prime}\right)$ which is birationally equivalent to $(f, X, C, L)$ such that $\left(f^{\prime}, X^{\prime}, C, L^{\prime}\right)$ satisfies one of the following conditions:

1. $K_{X^{\prime}}+2 L^{\prime}$ is $f^{\prime}$-nef,

2. $\left(f^{\prime}, X^{\prime}, C, L^{\prime}\right)$ is a scroll,

where $X^{\prime}$ is a normal projective variety with only $\mathbb{Q}$-factorial terminal singularities.

Proof. See Theorem 1.3 in [Fk3]. 
Theorem 1.6. Let $(f, X, C, L)$ be a quasi-polarized fiber space with $\operatorname{dim} X=3$ and $\operatorname{dim} C=1$. Then $g(L) \geq g(C)$.

Proof. See Theorem 1.4 in [Fk3].

Remark 1.7. If the Flip Conjecture (see $[\mathrm{KMM}]$ ) is true for $n=\operatorname{dim} X$, then Theorems 1.4, 1.5, and 1.6 are true for $n=\operatorname{dim} X$.

Lemma 1.8. Let $(f, X, Y, L)$ be a quasi-polarized fiber space, where $X$ is a normal projective variety of $\operatorname{dim} X \geq 2$ with only $\mathbb{Q}$-factorial canonical singularities. Assume that $K_{X / Y}+t L$ is $f$-nef, where $t$ is a positive integer. Then $\left(K_{X / Y}+t L\right) L^{n-1} \geq 0$.

Moreover if $\operatorname{dim} Y=1$, then $K_{X / Y}+t L$ is nef.

Proof. See Lemma 0.2 in [Fk3].

Definition 1.9. Let $X$ and $Y$ be $n$-dimensional projective manifolds, $L$ an ample divisor on $X$, and $\pi: X \rightarrow Y$ one point blowing up. Let $E$ be a $\pi$-exceptional reduced divisor. Then $\pi$ is said to be simple blowing up if $\left(E, L_{E}\right) \cong\left(\mathbb{P}^{n-1}, \mathcal{O}(1)\right)$.

Theorem 1.10. Let $(X, L)$ be a polarized manifold with $\operatorname{dim} X=n$. If $K_{X}+$ $(n-1) L$ is not nef, then $(X, L)$ is one of the following types:

(1) $\Delta(L)=0$, where $\Delta(L)=n+L^{n}-h^{0}(L)$. (See [Fj0].)

(2) $(X, L)$ is a scroll over a curve.

Proof. See $[\mathrm{Fj} 1]$ or $[\mathrm{I}]$.

Remark 1.11. Let $(X, L)$ be a polarized manifold with $\operatorname{dim} X=n$. If $K_{X}+(n-1) L$ is not nef, then $g(L) \geq q(X)$.

Theorem 1.12. Let $(X, L)$ be a polarized manifold with $\operatorname{dim} X=n \geq 3$. Assume that $K_{X}+(n-1) L$ is nef. If $K_{X}+(n-2) L$ is not nef, then $(X, L)$ is one of the following types:

(a) $(X, L)$ is obtained by some simple blowing up of another polarized manifold.

(b0) $(X, L)$ is a Del Pezzo manifold with $b_{2}(X)=1$, or $\left(\mathbb{P}^{3}, \mathcal{O}(j)\right)$ with $j=2$ or 3 , $\left(\mathbb{P}^{4}, \mathcal{O}(2)\right)$, or a hyperquadric in $\mathbb{P}^{4}$ with $L=\mathcal{O}(2)$.

(b1) There is a fibration $\Phi: X \rightarrow W$ over a curve $W$ with one of the following properties:

(b1-v) $\left(F, L_{F}\right) \cong\left(\mathbb{P}^{2}, \mathcal{O}(2)\right)$ for any fiber $F$ of $\Phi$.

(b1-q) Every fiber $F$ of $\Phi$ is an irreducible hyperquadric in $\mathbb{P}^{n}$ having only isolated singularities.

(b2) $(X, L)$ is a scroll over a smooth surface.

Proof. See [Fj1] or [I].

Theorem 1.13. Let $(X, L)$ be a quasi-polarized manifold. If $L$ is ample or $\operatorname{dim} X \leq$ 3 , then $g(L) \geq 0$.

Proof. See [Fj1], [Fj2], or [I].

Remark 1.14. If the Flip Conjecture (see $[\mathrm{KMM}]$ ) is true for $n=\operatorname{dim} X$, then Theorem 1.13 is true for $n=\operatorname{dim} X$.

Lemma 1.15. Let $(X, L)$ be a quasi-polarized manifold. If $\Delta(L)=0$, then $q(X)=$ 0 . 
Proof. By Theorem (1.1) in [Fj2], there exist a variety $W$, a birational morphism $f: X \rightarrow W$, and a very ample line bundle $H$ on $W$ such that $L=f^{*} H$ and $\Delta(H)=0$. Then $W$ is normal and has only rational singularities (see Corollary (5.17) in [Fj0]). Since $X$ is smooth, we have $q(X)=h^{1}\left(\mathcal{O}_{W}\right)$. But $h^{1}\left(\mathcal{O}_{W}\right)=0$ in this case (see Chapter I in $[\mathrm{Fj} 0]$ ). Hence $q(X)=0$.

Theorem 1.16. Let $(X, L)$ be a quasi-polarized manifold. If $L$ is ample or $\operatorname{dim} X \leq$ 3 , then $\Delta(L)=0$ if and only if $g(L)=0$.

Proof. See [Fj1] and [Fj2].

Definition 1.17. Let $(X, L)$ be a quasi-polarized surface. We say that $(X, L)$ is $L$-minimal if $L . E>0$ for any $(-1)$-curve $E$ on $X$. For any quasi-polarized surface $(X, L)$, there exists a birational morphism $\rho:(X, L) \rightarrow\left(X_{0}, L_{0}\right)$ such that $L=\rho^{*} L_{0}$ and $\left(X_{0}, L_{0}\right)$ is $L_{0}$-minimal. Then we call $\left(X_{0}, L_{0}\right)$ an $L$-minimalization of $(X, L)$.

Theorem 1.18 (classical). Let $(X, L)$ be a polarized manifold with $\operatorname{dim} X=n$. Assume that $|L|$ has no base point. If $g(L)=q(X)$, then $(X, L)$ is one of the following types.

(1) $\Delta(L)=0$.

(2) $(X, L)$ is a scroll over a curve.

Proof. See $[\mathrm{S}]$ or $[\mathrm{S}-\mathrm{V}]$.

Theorem 1.19. Let $(X, L)$ be a polarized manifold with $\operatorname{dim} X=n$. Assume that $|L|$ has no base point. Then $g(L) \geq 2 q(X)-1$ unless $(X, L)$ is a scroll over a curve $C$ with $g(C) \geq 2$.

Proof. We take $(n-2)$ general elements of $|L|$. By cutting these elements, there exists a polarized surface $\left(S, L_{S}\right)$ such that $g(L)=g\left(L_{S}\right)$ and $q(X)=q(S)$. So we may consider $\left(S, L_{S}\right)$.

(1) The case in which $\kappa(S) \geq 0$.

Then $g\left(L_{S}\right) \geq 2 q(S)-1$ by Corollary 3.2 in [Fk4]. Hence $g(L)=g\left(L_{S}\right) \geq$ $2 q(S)-1=2 q(X)-1$.

(2) The case in which $\kappa(S)=-\infty$.

If $q(S)=0$, then $g(L)=g\left(L_{S}\right) \geq 0=2 q(S)=2 q(X)$. So we may assume $q(S) \geq 1$. Then $K_{S}^{2} \leq 8(1-q(S))$. On the other hand,

$$
\begin{aligned}
\left(K_{S}+L\right)^{2} & =K_{S}^{2}+2\left(K_{S}+L_{S}\right) L_{S}-L_{S}^{2} \\
& \leq 8(1-q(S))+4\left(g\left(L_{S}\right)-1\right)-L_{S}^{2} \\
& =4\left(g\left(L_{S}\right)-2 q(S)+1\right)-L_{S}^{2} .
\end{aligned}
$$

If $K_{S}+L_{S}$ is nef, then $\left(K_{S}+L_{S}\right)^{2} \geq 0$. So we have $g\left(L_{S}\right) \geq 2 q(S)$.

If $K_{S}+L_{S}$ is not nef, then $\left(S, L_{S}\right)$ is a scroll over a curve by Mori theory (see [Fk1]). Hence $g(L)=g\left(L_{S}\right) \geq 2 q(S)=2 q(X)$ unless $\left(S, L_{S}\right)$ is a scroll over a curve. On the other hand, if $\left(S, L_{S}\right)$ is a scroll over a curve with $q(S) \geq 1$, then $(X, L)$ is a scroll over a curve $C$ by Bădescu ([B1], [B2], [B3]). (See also (5.5) in $[\mathrm{B}-\mathrm{S}]$. 1.19 .

If $g(C) \leq 1$, then $g(L) \geq 2 q(X)-1$. This completes the proof of Theorem 
Notation 1.20. Let $D$ be an effective divisor on a smooth projective variety $X$ and $D=\sum_{i} a_{i} D_{i}$ its prime decomposition, where $a_{i} \geq 1$ for any $i$.

Then we write $D_{\text {red }}=\sum_{i} D_{i}$.

\section{MAin Results}

In this section we consider (quasi-)polarized 3-folds with $h^{0}(L) \geq 2$.

Theorem 2.1. Let $(X, L)$ be a quasi-polarized 3-fold with $h^{0}(L) \geq 2$. Then $g(L) \geq$ $q(X)$.

Proof. By Theorem 1.4, there exists a quasi-polarized variety $\left(X^{\prime}, L^{\prime}\right)$ which is birationally equivalent to $(X, L)$ and satisfies one of the following conditions:

1. $K_{X^{\prime}}+2 L^{\prime}$ is nef for the canonical $\mathbb{Q}$-bundle $K_{X^{\prime}}$,

2. $\Delta\left(L^{\prime}\right)=0$,

3. $\left(X^{\prime}, L^{\prime}\right)$ is a scroll over a curve,

where $X^{\prime}$ is a normal projective variety with only $\mathbb{Q}$-factorial terminal singularities.

Since $g(L)=g\left(L^{\prime}\right)$ and $q(X)=q\left(X^{\prime}\right)$, we may assume that $X$ has only $\mathbb{Q}$ factorial terminal singularities and satisfies one of the above conditions.

If $(X, L)$ is the type (2), then $q(X)=0$ by Lemma 1.15 . Since $g(L) \geq 0$ for any quasi-polarized 3 -fold by Theorem 1.13, we obtain that $g(L) \geq q(X)$ in this case.

If $(X, L)$ is the type (3), then $g(L)=q(X)$ by easy calculation.

So we may assume that $K_{X}+2 L$ is nef. Let $\pi: \widetilde{X} \rightarrow X$ be a resolution of $X$ such that $\widetilde{X} \backslash \pi^{-1}(\operatorname{Sing}(X)) \cong X \backslash \operatorname{Sing}(X)$, and $\widetilde{L}=\pi^{*}(L)$. Then $h^{0}(L)=h^{0}(\widetilde{L}) \geq 2$. Let $\Lambda$ be a linear pencil which is contained in $|\widetilde{L}|$ such that $\Lambda=\Lambda_{M}+Z$, where $\Lambda_{M}$ is the movable part of $\Lambda$ and $Z$ is the fixed part of $|\widetilde{L}|$. We will make a fiber space by using this $\Lambda$. Let $\varphi: \widetilde{X} \rightarrow \mathbb{P}^{1}$ be the rational map associated with $\Lambda_{M}$, and $\theta: \widetilde{X}^{\prime} \rightarrow \widetilde{X}$ an elimination of indeterminacy of $\varphi$. So we obtain a surjective morphism $\varphi^{\prime}: \widetilde{X}^{\prime} \rightarrow \mathbb{P}^{1}$. If necessary, we take the Stein factorization $\delta: C \rightarrow \mathbb{P}^{1}$ of $\varphi^{\prime}$. Then we have a fiber space $f^{\prime}: \widetilde{X}^{\prime} \rightarrow C$ such that $\varphi^{\prime}=\delta \circ f^{\prime}$. We consider this quasi-polarized fiber space $\left(f^{\prime}, \widetilde{X}^{\prime}, C, \theta^{*} \widetilde{L}\right)$.

Case (1). $g(C) \geq 1$. In this case, $\theta=$ id. So $\tilde{X}^{\prime}=\tilde{X}$. Then

$$
g(L)=g(\widetilde{L})=g(C)+\frac{1}{2}\left(K_{\widetilde{X} / C}+2 \widetilde{L}\right)(\widetilde{L})^{2}+\left(\widetilde{L}^{2} F^{\prime}-1\right)(g(C)-1),
$$

where $F^{\prime}$ is a general fiber of $f^{\prime}$.

By Theorem 1.5, there exists a quasi-polarized fiber space $\left(f_{1}, X_{1}, C, L_{1}\right)$ which is birationally equivalent to $\left(f^{\prime}, \widetilde{X}, C, \widetilde{L}\right)$ such that $\left(f_{1}, X_{1}, C, L_{1}\right)$ satisfies one of the following conditions:

1. $K_{X_{1}}+2 L_{1}$ is $f_{1}$-nef,

2. $\left(f_{1}, X_{1}, C, L_{1}\right)$ is a scroll,

where $X_{1}$ has a normal projective variety with only $\mathbb{Q}$-factorial terminal singularities.

If $\left(f_{1}, X_{1}, C, L_{1}\right)$ is the type (2), then $g(L)=g\left(L_{1}\right)=g(C)=q\left(X_{1}\right)=q(X)$. So we may assume that $K_{X_{1}}+2 L_{1}$ is $f_{1}$-nef. Then by Lemma $1.8,\left(K_{X_{1} / C}+2 L_{1}\right)$ is nef. We take a general member $B$ of $|\widetilde{L}|$. Then $B \equiv a F^{\prime}+Z$, where $F^{\prime}$ is a general fiber of $f^{\prime}$ and $a=\operatorname{deg} \delta$. 
Claim 2.2. $\left(K_{\widetilde{X} / C}+2 \widetilde{L}\right) \widetilde{L}\left(\widetilde{L}-t F^{\prime}\right) \geq 0$ for any natural number $t \leq a$.

Proof. By Theorem 1.5, there exist a smooth projective variety $G$ and birational morphisms $\varepsilon_{1}: G \rightarrow \widetilde{X}$ and $\varepsilon_{2}: G \rightarrow X_{1}$ such that $\varepsilon_{1}^{*}(\widetilde{L})=\varepsilon_{2}^{*}\left(L_{1}\right)$.

Then

$$
\begin{aligned}
& \left(K_{\widetilde{X} / C}+2 \widetilde{L}\right) \widetilde{L}\left(\widetilde{L}-t F^{\prime}\right) \\
& =\left(K_{G / C}+2 \varepsilon_{1}^{*}(\widetilde{L})\right)\left(\varepsilon_{1}^{*}(\widetilde{L})\right)\left(\varepsilon_{1}^{*}\left(\widetilde{L}-t F^{\prime}\right)\right) \\
& =\left(\varepsilon_{2}^{*}\left(K_{X_{1} / C}+2 L_{1}\right)+E_{\varepsilon_{2}}\right)\left(\varepsilon_{2}^{*} L_{1}\right)\left(\varepsilon_{2}^{*}\left(L_{1}-t F_{1}\right)\right) \\
& =\varepsilon_{2}^{*}\left(K_{X_{1} / C}+2 L_{1}\right)\left(\varepsilon_{2}^{*} L_{1}\right)\left(\varepsilon_{2}^{*}\left(L_{1}-t F_{1}\right)\right),
\end{aligned}
$$

where $E_{\varepsilon_{2}}$ is an $\varepsilon_{2}$-exceptional $\mathbb{Q}$-divisor and $F_{1}$ is a general fiber of $f_{1}$.

We remark that $\varepsilon_{1}^{*}\left(\widetilde{L}-t F^{\prime}\right)=\varepsilon_{2}^{*}\left(L_{1}-t F_{1}\right), \varepsilon_{1}^{*}\left(\widetilde{L}-t F^{\prime}\right)$ is numerically equivalent to an effective divisor, and $K_{X_{1} / C}+2 L_{1}$ is nef. Hence we obtain

$$
\varepsilon_{2}^{*}\left(K_{X_{1} / C}+2 L_{1}\right)\left(\varepsilon_{2}^{*} L_{1}\right)\left(\varepsilon_{2}^{*}\left(L_{1}-t F_{1}\right)\right) \geq 0 .
$$

This completes the proof of this Claim.

Therefore

$$
g(\widetilde{L}) \geq g(C)+\left(K_{\widetilde{X} / C}+2 \widetilde{L}\right) \widetilde{L} F^{\prime}
$$

since $\widetilde{L}^{2} F^{\prime} \geq 1, g(C) \geq 1$, and $a \geq 2$. So we obtain

$$
\begin{aligned}
g(\widetilde{L}) & \geq g(C)+\left(K_{F^{\prime}}+2 \widetilde{L}_{F^{\prime}}\right) \widetilde{L}_{F^{\prime}} \\
& =g(C)+\left(K_{F^{\prime}}+\widetilde{L}_{F^{\prime}}\right) \widetilde{L}_{F^{\prime}}+\widetilde{L}_{F^{\prime}}^{2} \\
& =g(C)+2 g\left(\widetilde{L}_{F^{\prime}}\right)+\widetilde{L}_{F^{\prime}}^{2}-2 \\
& =g(C)+g\left(\widetilde{L}_{F^{\prime}}\right)+g\left(\widetilde{L}_{F^{\prime}}\right)+\widetilde{L}_{F^{\prime}}^{2}-2 .
\end{aligned}
$$

Since $h^{0}\left(\widetilde{L}_{F^{\prime}}\right) \geq 1$ and $\operatorname{dim} F^{\prime}=2$, we have $g\left(\widetilde{L}_{F^{\prime}}\right) \geq q\left(F^{\prime}\right)$. Hence

$$
\begin{aligned}
g(\widetilde{L}) & \geq g(C)+q\left(F^{\prime}\right)+g\left(\widetilde{L}_{F^{\prime}}\right)+\widetilde{L}_{F^{\prime}}^{2}-2 \\
& \geq q(\widetilde{X})+g\left(\widetilde{L}_{F^{\prime}}\right)+\widetilde{L}_{F^{\prime}}^{2}-2 .
\end{aligned}
$$

If $g\left(\widetilde{L}_{F^{\prime}}\right)=0$, then $q\left(F^{\prime}\right)=0$ and $q(\widetilde{X})=g(C)$. On the other hand, $g(\widetilde{L}) \geq g(C)$ by Theorem 1.6. So $g(L)=g(\widetilde{L}) \geq g(C)=q(\widetilde{X})=q(X)$.

If $g\left(\widetilde{L}_{F^{\prime}}\right) \geq 1$, then $g(\widetilde{L}) \geq q(\widetilde{X})$ since $\widetilde{L}_{F^{\prime}}^{2} \geq 1$.

Case (2). $g(C)=0$. Let $\widetilde{D}=\theta\left(F^{\prime}\right)$ and $D=\pi(\widetilde{D})$. We remark that $D \neq 0$ because $F^{\prime}$ is a general fiber. Then $\widetilde{L}-\widetilde{D}$ is linearly equivalent to an effective divisor. We put $\gamma=\pi \circ \theta$. Then

$$
\begin{aligned}
g(L)=g(\widetilde{L})=g\left(\theta^{*} \widetilde{L}\right) & =1+\frac{1}{2}\left(K_{\widetilde{X}^{\prime}}+2 \theta^{*} \widetilde{L}\right)\left(\theta^{*} \widetilde{L}\right)^{2} \\
& =1+\frac{1}{2}\left(\theta^{*}\left(K_{\widetilde{X}}+2 \widetilde{L}\right)\right)\left(\theta^{*} \widetilde{L}\right)^{2} \\
& =1+\frac{1}{2}\left(\theta^{*}\left(K_{\widetilde{X}}\right)+2 \gamma^{*} L\right)\left(\theta^{*} \widetilde{L}\right)^{2} \\
& =1+\frac{1}{2} \gamma^{*}\left(K_{X}+2 L\right)\left(\theta^{*} \widetilde{L}\right)^{2} \\
& \geq 1+\frac{1}{2} \gamma^{*}\left(K_{X}+2 L\right)\left(\theta^{*} \widetilde{L}\right) F^{\prime}
\end{aligned}
$$

because $K_{X}+2 L$ is nef. 
Let $K_{\widetilde{X}}=\pi^{*}\left(K_{X}\right)+\sum a_{i} \widetilde{E}_{i}$, where $\widetilde{E}_{i}$ is a $\pi$-exceptional effective divisor.

Claim 2.3. $\left(\theta^{*} \widetilde{E}_{i}\right)\left(\theta^{*} \widetilde{L}\right)\left(F^{\prime}\right)=0$.

Proof.

$$
\begin{aligned}
\left(\theta^{*} \widetilde{E}_{i}\right)\left(\theta^{*} \widetilde{L}\right)\left(F^{\prime}\right) & =\left(\widetilde{E}_{i}\right)(\widetilde{L}) \widetilde{D} \\
& =\left(\left.\widetilde{E}_{i}\right|_{\widetilde{D}}\right)\left(\left.\pi\right|_{\widetilde{D}}\right)^{*}\left(\left.L\right|_{D}\right) .
\end{aligned}
$$

By the definition of $\pi, \pi\left(\widetilde{E}_{i}\right) \subset \operatorname{Sing}(X)$. On the other hand, $\operatorname{Codim} \operatorname{Sing}(X) \geq 3$ because $X$ has only terminal singularities. Hence $\operatorname{dim}\left(\left.\pi\right|_{\tilde{D}}\right)\left(\widetilde{E}_{i}\right)$ is at most 0 in $D$. We remark that $\operatorname{dim} D=2$. Therefore $\left(\left.\widetilde{E}_{i}\right|_{\widetilde{D}}\right)\left(\left.\pi\right|_{\widetilde{D}}\right)^{*}\left(\left.L\right|_{D}\right)=0$. This completes the proof of Claim 2.3.

Hence we obtain

$$
\begin{aligned}
g(L) & \geq 1+\frac{1}{2} \theta^{*}\left(\pi^{*} K_{X}+2 \widetilde{L}\right)\left(\theta^{*} \widetilde{L}\right) F^{\prime} \\
& =1+\frac{1}{2} \theta^{*}\left(\pi^{*} K_{X}+\sum a_{i} \widetilde{E}_{i}+2 \widetilde{L}\right)\left(\theta^{*} \widetilde{L}\right) F^{\prime} \\
& =1+\frac{1}{2} \theta^{*}\left(K_{\widetilde{X}}+2 \widetilde{L}\right)\left(\theta^{*} \widetilde{L}\right) F^{\prime} .
\end{aligned}
$$

Since $F^{\prime}$ is nef and $\widetilde{L}-\widetilde{D}$ is linearly equivalent to an effective divisor, we obtain

$$
\begin{aligned}
g(L) & \geq 1+\frac{1}{2} \theta^{*}\left(K_{\widetilde{X}}+2 \widetilde{L}\right)\left(\theta^{*} \widetilde{L}\right) F^{\prime} \\
& =1+\frac{1}{2}\left(\theta^{*}\left(K_{\widetilde{X}}\right)+\theta^{*}(\widetilde{D})+\theta^{*}(\widetilde{L}-\widetilde{D})+\theta^{*} \widetilde{L}\right)\left(\theta^{*} \widetilde{L}\right) F^{\prime} \\
& \geq 1+\frac{1}{2}\left(\theta^{*}\left(K_{\widetilde{X}}+\widetilde{D}\right)+\theta^{*} \widetilde{L}\right)\left(\theta^{*} \widetilde{L}\right) F^{\prime} .
\end{aligned}
$$

Let $\theta_{i}: X_{i} \rightarrow X_{i-1}$ be a blowing up at a smooth center $B_{i-1}$ and $\theta=\theta_{1} \circ \cdots \circ \theta_{t}$. Let $X_{0}=\widetilde{X}$ and $X_{t}=\widetilde{X}^{\prime}$. Let $D_{i}$ be the strict transform of $D_{i-1}, D_{0}=\widetilde{D}$, and $D_{t}=F^{\prime}$. Let $\theta_{i}^{*} D_{i-1}=D_{i}+d_{i} E_{i}$, where $E_{i}$ is a $\theta_{i}$-exceptional effective reduced divisor. Then $K_{X_{i}}=\theta_{i}^{*}\left(K_{X_{i-1}}\right)+a_{i} E_{i}$, where $a_{i}=1$ if $\operatorname{dim} B_{i-1}=1$ and $a_{i}=2$ if $\operatorname{dim} B_{i-1}=0$.

\section{Claim 2.4.}

$$
\left(\theta_{t}^{*} \circ \cdots \circ \theta_{k}^{*}\right)\left(K_{X_{k-1}}+D_{k-1}\right)\left(\theta^{*} \widetilde{L}\right) F^{\prime} \geq\left(\theta_{t}^{*} \circ \cdots \circ \theta_{k+1}^{*}\right)\left(K_{X_{k}}+D_{k}\right)\left(\theta^{*} \widetilde{L}\right) F^{\prime} .
$$

Proof. (1) The case in which $\operatorname{dim} B_{k-1}=0$.

In this case

$$
\begin{aligned}
& \left(\theta_{t}^{*} \circ \cdots \circ \theta_{k+1}^{*}\right)\left(E_{k}\right)\left(\theta^{*} \widetilde{L}\right) F^{\prime} \\
& =\left(E_{k}\right)\left(\left(\theta_{k}^{*} \circ \cdots \circ \theta_{1}^{*}\right)(\widetilde{L})\right) D_{k} \\
& =\left(\left.E_{k}\right|_{D_{k}}\right)\left(\left.\theta_{k}\right|_{D_{k}}\right)^{*}\left(\left.\left(\theta_{k-1}^{*} \circ \cdots \circ \theta_{1}^{*} \widetilde{L}\right)\right|_{D_{k-1}}\right) \\
& =0 .
\end{aligned}
$$


Hence

$$
\begin{aligned}
& \left(\theta_{t}^{*} \circ \cdots \circ \theta_{k}^{*}\right)\left(K_{X_{k-1}}+D_{k-1}\right)\left(\theta^{*} \widetilde{L}\right) F^{\prime} \\
& =\left(\theta_{t}^{*} \circ \cdots \circ \theta_{k+1}^{*}\right)\left(\theta_{k}^{*}\left(K_{X_{k-1}}\right)+\theta_{k}^{*} D_{k-1}\right)\left(\theta^{*} \widetilde{L}\right) F^{\prime} \\
& =\left(\theta_{t}^{*} \circ \cdots \circ \theta_{k+1}^{*}\right)\left(\theta_{k}^{*}\left(K_{X_{k-1}}\right)+2 E_{k}+D_{k}\right)\left(\theta^{*} \widetilde{L}\right) F^{\prime} \\
& =\left(\theta_{t}^{*} \circ \cdots \circ \theta_{k+1}^{*}\right)\left(K_{X_{k}}+D_{k}\right)\left(\theta^{*} \widetilde{L}\right) F^{\prime} .
\end{aligned}
$$

(2) The case in which $\operatorname{dim} B_{k-1}=1$.

In this case, $\left(\theta_{t}^{*} \circ \cdots \circ \theta_{k+1}^{*}\right)\left(E_{k}\right)\left(\theta^{*} \widetilde{L}\right) F^{\prime} \geq 0$ since $F^{\prime}$ is nef. In particular, $\left(\theta_{t}^{*} \circ \cdots \circ \theta_{k+1}^{*}\right)\left(E_{k}\right)\left(\theta^{*} \widetilde{L}\right) F^{\prime}=0$ if $d_{k}=0$ (that is, $D_{k}=\theta_{k}^{*}\left(D_{k-1}\right)$ ) because

$$
\begin{aligned}
& \left(\theta_{t}^{*} \circ \cdots \circ \theta_{k+1}^{*}\right)\left(E_{k}\right)\left(\theta^{*} \widetilde{L}\right) F^{\prime} \\
& =\left(E_{k}\right)\left(\left(\theta_{k}^{*} \circ \cdots \circ \theta_{1}^{*}\right)(\widetilde{L})\right) D_{k} \\
& =\left(E_{k}\right)\left(\left(\theta_{k}^{*} \circ \cdots \circ \theta_{1}^{*}\right)(\widetilde{L})\right) \theta_{k}^{*} D_{k-1} \\
& =0 .
\end{aligned}
$$

Hence by the above argument

$$
\begin{aligned}
& \left(\theta_{t}^{*} \circ \cdots \circ \theta_{k}^{*}\right)\left(K_{X_{k-1}}+D_{k-1}\right)\left(\theta^{*} \widetilde{L}\right) F^{\prime} \\
& =\left(\theta_{t}^{*} \circ \cdots \circ \theta_{k+1}^{*}\right)\left(\theta_{k}^{*}\left(K_{X_{k-1}}\right)+D_{k}+d_{k} E_{k}\right)\left(\theta^{*} \widetilde{L}\right) F^{\prime} \\
& \geq\left(\theta_{t}^{*} \circ \cdots \circ \theta_{k+1}^{*}\right)\left(\theta_{k}^{*}\left(K_{X_{k-1}}\right)+E_{k}+D_{k}\right)\left(\theta^{*} \widetilde{L}\right) F^{\prime} \\
& \geq\left(\theta_{t}^{*} \circ \cdots \circ \theta_{k+1}^{*}\right)\left(K_{X_{k}}+D_{k}\right)\left(\theta^{*} \widetilde{L}\right) F^{\prime} .
\end{aligned}
$$

This completes the proof of this Claim.

By Claim 2.4,

$$
\begin{aligned}
g(L) & \geq 1+\frac{1}{2}\left(\theta^{*}\left(K_{\widetilde{X}}+\widetilde{D}\right)+\theta^{*} \widetilde{L}\right)\left(\theta^{*} \widetilde{L}\right) F^{\prime} \\
& \geq 1+\frac{1}{2}\left(K_{\widetilde{X}^{\prime}}+F^{\prime}+\theta^{*} \widetilde{L}\right)\left(\theta^{*} \widetilde{L}\right) F^{\prime} \\
& =g\left(\left.\theta^{*} \widetilde{L}\right|_{F^{\prime}}\right) .
\end{aligned}
$$

Since $h^{0}\left(\left.\theta^{*} \widetilde{L}\right|_{F^{\prime}}\right) \geq 1$ and $\operatorname{dim} F^{\prime}=2$, we have $g\left(\left.\theta^{*} \widetilde{L}\right|_{F^{\prime}}\right) \geq q\left(F^{\prime}\right)$. On the other hand, $q\left(F^{\prime}\right) \geq q\left(\widetilde{X}^{\prime}\right)=q(X)$ in this case. Therefore $g(L) \geq g\left(\left.\theta^{*} \widetilde{L}\right|_{F^{\prime}}\right) \geq q\left(F^{\prime}\right) \geq$ $q(X)$. This completes the proof of Theorem 2.1.

By the same argument as the proof of Theorem 2.1, we can prove the following Theorem.

Theorem 2.5. Let $(X, L)$ be a quasi-polarized manifold with $\operatorname{dim} X=n \geq 4$ and $h^{0}(L) \geq 2$. Assume that the Flip Conjecture (see $\left.[\mathrm{KMM}]\right)$ is true and $(X, L)$ is not birationally equivalent to a quasi-polarized variety $\left(X^{\prime}, L^{\prime}\right)$ such that $\Delta\left(L^{\prime}\right)=0$ or $\left(X^{\prime}, L^{\prime}\right)$ is a scroll over a curve, where $X^{\prime}$ is a normal projective variety with only $\mathbb{Q}$-factorial terminal singularities. Then there exists a quasi-polarized fiber space $(f, Y, C, A)$ with $\operatorname{dim} Y=n, \operatorname{dim} C=1$, and $h^{0}(A) \geq 2$ such that $Y$ is birationally equivalent to $X, g(L) \geq g\left(\left.A\right|_{F}\right)+g(C)$ and $q(F)+g(C) \geq q(Y)=q(X)$, where $F$ is a general fiber of $f$.

We fix the notation which is used in the following theorems. 
Notation 2.6. Let $(X, L)$ be a polarized manifold with $\operatorname{dim} X=n \geq 3$ and $h^{0}(L) \geq 2$. Let $\Lambda$ be a linear pencil which is contained in $|L|$ such that $\Lambda=\Lambda_{M}+Z$, where $\Lambda_{M}$ is the movable part of $\Lambda$ and $Z$ is the fixed part of $|L|$. We will make a fiber space by using this $\Lambda$. Let $\varphi: X \rightarrow \mathbb{P}^{1}$ be the rational map associated with $\Lambda_{M}$ and $\theta: X^{\prime} \rightarrow X$ be an elimination of indeterminacy of $\varphi$. So we obtain a surjective morphism $\varphi^{\prime}: X^{\prime} \rightarrow \mathbb{P}^{1}$. If necessary, we take the Stein factorization $\delta: C \rightarrow \mathbb{P}^{1}$ of $\varphi^{\prime}$. Then we have a fiber space $f^{\prime}: X^{\prime} \rightarrow C$ such that $\varphi^{\prime}=\delta \circ f^{\prime}$. Let $a=\operatorname{deg} \delta$ and $F^{\prime}$ be a general fiber of $f^{\prime}$.

We remark that we can prove the following Theorem for a polarized manifold $(X, L)$ of $\operatorname{dim} X=n \geq 4$.

Theorem 2.7. Let $(X, L)$ be a polarized manifold with $\operatorname{dim} X=n \geq 4$ and $h^{0}(L) \geq$ 2. We use Notation 2.6. Then

$$
g(L) \geq g(C)+g\left(\theta^{*} L_{F^{\prime}}\right)
$$

unless $\Delta(L)=0$ or $(X, L)$ is a scroll over a curve.

Proof. Case $(a): g(C) \geq 1$.

Then we remark that $\theta=$ id. We consider the polarized fiber space $\left(f^{\prime}, X, C, L\right)$. Then

$$
g(L)=g(C)+\frac{1}{2}\left(K_{X / C}+(n-1) L\right) L^{n-1}+\left(L^{n-1} F^{\prime}-1\right)(g(C)-1),
$$

where $F^{\prime}$ is a general fiber of $f^{\prime}$.

If $K_{X / C}+(n-1) L$ is not nef, then $\left(f^{\prime}, X, C, L\right)$ is scroll by Theorem 1.3. Hence we may assume that $K_{X / C}+(n-1) L$ is nef. Since $a \geq 2, L-2 F^{\prime}$ is numerically equivalent to an effective divisor. Hence $g(L) \geq g(C)+\left(K_{X / C}+(n-1) L\right) L^{n-2} F^{\prime}$ since $\left(L_{F^{\prime}}\right)^{n-1} \geq 1$.

So we have

$$
\begin{aligned}
g(L) & \geq g(C)+\left(K_{F^{\prime}}+(n-1) L_{F^{\prime}}\right)\left(L_{F^{\prime}}\right)^{n-2} \\
& =g(C)+\left(K_{F^{\prime}}+(n-2) L_{F^{\prime}}\right)\left(L_{F^{\prime}}\right)^{n-2}+\left(L_{F^{\prime}}\right)^{n-1} \\
& =g(C)+2 g\left(L_{F^{\prime}}\right)-2+\left(L_{F^{\prime}}\right)^{n-1} \\
& =g(C)+g\left(L_{F^{\prime}}\right)+g\left(L_{F^{\prime}}\right)-2+\left(L_{F^{\prime}}\right)^{n-1} .
\end{aligned}
$$

If $g\left(L_{F^{\prime}}\right) \geq 1$, then $g(L) \geq g(C)+g\left(L_{F^{\prime}}\right)$. So we assume that $g\left(L_{F^{\prime}}\right)=0$. By Theorem 1.3, $g(L) \geq g(C)=g(C)+g\left(L_{F^{\prime}}\right)$.

Case (b): $g(C)=0$. Let $D$ be an irreducible reduced divisor such that the strict transform of $D$ by $\theta$ is a general fiber $F^{\prime}$. Then $L-D$ is linearly equivalent to an effective divisor. We may assume that $K_{X}+(n-1) L$ is nef by Theorem 1.10.

So we have

$$
\begin{aligned}
g(L)=g\left(\theta^{*} L\right) & =1+\frac{1}{2}\left(K_{X^{\prime}}+(n-1) \theta^{*} L\right)\left(\theta^{*} L\right)^{n-1} \\
& =1+\frac{1}{2} \theta^{*}\left(K_{X}+(n-1) L\right)\left(\theta^{*} L\right)^{n-1} \\
& \geq 1+\frac{1}{2} \theta^{*}\left(K_{X}+(n-1) L\right)\left(\theta^{*} L\right)^{n-2} F^{\prime} \\
& =1+\frac{1}{2}\left(\theta^{*}\left(K_{X}+D\right)+\theta^{*}(L-D)+(n-2) \theta^{*} L\right)\left(\theta^{*} L\right)^{n-2} F^{\prime}
\end{aligned}
$$


Since $\theta^{*}(L-D)\left(\theta^{*} L\right)^{n-2} F^{\prime} \geq 0$, we have

$$
g(L) \geq 1+\frac{1}{2}\left(\theta^{*}\left(K_{X}+D\right)+(n-2) \theta^{*} L\right)\left(\theta^{*} L\right)^{n-2} F^{\prime} .
$$

By the same argument as in the proof of Claim 2.4, we can prove

$$
\theta^{*}\left(K_{X}+D\right)\left(\theta^{*} L\right)^{n-2} F^{\prime} \geq\left(K_{X^{\prime}}+F^{\prime}\right)\left(\theta^{*} L\right)^{n-2} F^{\prime} .
$$

Hence

$$
\begin{aligned}
g(L) & \geq 1+\frac{1}{2}\left(K_{X^{\prime}}+F^{\prime}+(n-2) \theta^{*} L\right)\left(\theta^{*} L\right)^{n-2} F^{\prime} \\
& =g\left(\theta^{*} L_{F^{\prime}}\right) .
\end{aligned}
$$

Before we study polarized 3-folds $(X, L)$ such that $g(L)=q(X)$, we prove the following Theorem.

Theorem 2.8. Let $(X, L)$ be a polarized 3-fold with $h^{0}(L) \geq 2$. We use Notation 2.6. Then

1. $g(L) \geq a q(X)$ if $g(C)=0$,

2. $g(L) \geq q(X)+(a-1) q\left(F^{\prime}\right)$ if $g(C) \geq 1$,

unless $\Delta(L)=0$ or $(X, L)$ is a scroll over a curve.

Proof. If $K_{X}+2 L$ is not nef, then $\Delta(L)=0$ or $(X, L)$ is a scroll over a curve by Theorem 1.10. So we may assume that $K_{X}+2 L$ is nef. Let $Z=\sum_{i=1}^{m} b_{i} Z_{i}$, and $Z_{i}^{\prime}$ be the strict transform of $Z_{i}$ by $\theta$. Let $\theta^{\prime}: X^{\prime \prime} \rightarrow X^{\prime}$ be a birational morphism such that $Z_{i}^{\prime \prime}$ is a smooth surface, where $Z_{i}^{\prime \prime}$ is the strict transform of $Z_{i}^{\prime}$ by $\theta^{\prime}$. We can take a general element $B \in|L|$ such that $B=G_{1}+\cdots+G_{a}+Z$, where each $G_{i}$ is the image of a general fiber of $f^{\prime}$ by $\theta$. Let $h=f^{\prime} \circ \theta^{\prime}$ and $\pi=\theta \circ \theta^{\prime}$. Then the strict transform of $G_{i}$ by $\theta \circ \theta^{\prime}$ is a general fiber of $h$. Let $\pi=\theta_{1} \circ \cdots \circ \theta_{u}$, where $\theta_{i}: X_{i} \rightarrow X_{i-1}$ is blowing up at smooth center $B_{i-1}$ on $X_{i-1}$. Let $F_{i}^{\prime \prime}$ (resp. $Z_{i}^{\prime \prime}$ ) be the strict transform of $G_{i}$ (resp. $Z_{i}$ ) by $\pi$. Then

$$
\begin{aligned}
g(L)=g\left(\pi^{*} L\right) & =1+\frac{1}{2}\left(K_{X^{\prime \prime}}+2 \pi^{*} L\right)\left(\pi^{*} L\right)^{2} \\
& =1+\frac{1}{2} \pi^{*}\left(K_{X}+2 L\right)\left(\pi^{*} L\right)\left(\pi^{*} B\right) \\
& \geq 1+\frac{1}{2} \pi^{*}\left(K_{X}+2 L\right)\left(\pi^{*} L\right)\left(\pi^{*}\left(B_{\text {red }}\right)\right) .
\end{aligned}
$$

Let $B_{\mathrm{nr}}=B-B_{\mathrm{red}}$.

Claim 2.9. $B_{\mathrm{nr}} B_{\mathrm{red}} L \geq 0$.

Proof. If $B_{\mathrm{nr}}=0$, then this is obvious. So we may assume $B_{\mathrm{nr}} \neq 0$. Since $L$ is ample, Bs $|n L|=\phi$ for large $n$. We can take a general element $S \in|n L|$ such that $S$ is smooth and $\left.B_{\mathrm{nr}}\right|_{S}$ and $\left.B_{\mathrm{red}}\right|_{S}$ are nonzero effective divisors. Since $L$ is ample, so is $L_{S}$. On the other hand, $L_{S}=\left.B_{\mathrm{nr}}\right|_{S}+\left.B_{\text {red }}\right|_{S}$. By Lemma 2 in [Ra], $\left.\left.B_{\mathrm{nr}}\right|_{S} B_{\mathrm{red}}\right|_{S}>0$. Therefore $B_{\mathrm{nr}} B_{\mathrm{red}} L>0$. This completes the proof of this Claim. 
So we obtain

$$
\begin{aligned}
g(L) \geq 1 & +\frac{1}{2} \pi^{*}\left(K_{X}+2 L\right)\left(\pi^{*} L\right)\left(\pi^{*}\left(B_{\mathrm{red}}\right)\right) \\
= & +\frac{1}{2}\left(\pi^{*}\left(K_{X}\right)+\pi^{*}\left(B_{\mathrm{red}}\right)+\pi^{*}\left(B_{\mathrm{nr}}\right)+\pi^{*} L\right)\left(\pi^{*} L\right)\left(\pi^{*}\left(B_{\mathrm{red}}\right)\right) \\
\geq & +\frac{1}{2}\left(\pi^{*}\left(K_{X}+B_{\mathrm{red}}\right)+\pi^{*} L\right)\left(\pi^{*} L\right)\left(\pi^{*}\left(B_{\mathrm{red}}\right)\right) \\
= & +\frac{1}{2} \sum_{i=1}^{a}\left(\pi^{*}\left(K_{X}+B_{\mathrm{red}}\right)+\pi^{*} L\right)\left(\pi^{*} L\right) F_{i}^{\prime \prime} \\
& +\frac{1}{2} \sum_{i=1}^{m}\left(\pi^{*}\left(K_{X}+B_{\mathrm{red}}\right)+\pi^{*} L\right)\left(\pi^{*} L\right) Z_{i}^{\prime \prime} \\
= & +\frac{1}{2} \sum_{i=1}^{a}\left(\pi^{*}\left(K_{X}+G_{i}\right)+\pi^{*} L\right)\left(\pi^{*} L\right) F_{i}^{\prime \prime} \\
& +\frac{1}{2} \sum_{i=1}^{m}\left(\pi^{*}\left(K_{X}+Z_{i}\right)+\pi^{*} L\right)\left(\pi^{*} L\right) Z_{i}^{\prime \prime} \\
& +\frac{1}{2} \sum_{i=1}^{a} \pi^{*}\left(B_{\mathrm{red}}-G_{i}\right)\left(\pi^{*} L\right) F_{i}^{\prime \prime}+\frac{1}{2} \sum_{i=1}^{m} \pi^{*}\left(B_{\mathrm{red}}-Z_{i}\right)\left(\pi^{*} L\right) Z_{i}^{\prime \prime} .
\end{aligned}
$$

Since $L$ is ample and $B$ is connected,

$$
\begin{aligned}
& \frac{1}{2}\left(\sum_{i=1}^{a} \pi^{*}\left(B_{\mathrm{red}}-G_{i}\right)\left(\pi^{*} L\right) F_{i}^{\prime \prime}+\sum_{i=1}^{m} \pi^{*}\left(B_{\mathrm{red}}-Z_{i}\right)\left(\pi^{*} L\right) Z_{i}^{\prime \prime}\right) \\
& =\frac{1}{2}\left(\left(B_{\mathrm{red}}\right)^{2}-\left(\sum_{i=1}^{a} G_{i}^{2}+\sum_{i=1}^{m} Z_{i}^{2}\right)\right) L \\
& \geq a+m-1 .
\end{aligned}
$$

Hence

$$
\begin{aligned}
g(L) \geq & 1+\frac{1}{2} \sum_{i=1}^{a}\left(\pi^{*}\left(K_{X}+G_{i}\right)+\pi^{*} L\right)\left(\pi^{*} L\right) F_{i}^{\prime \prime} \\
& +\frac{1}{2} \sum_{i=1}^{m}\left(\pi^{*}\left(K_{X}+Z_{i}\right)+\pi^{*} L\right)\left(\pi^{*} L\right) Z_{i}^{\prime \prime} \\
& +(a+m-1) \\
= & \sum_{i=1}^{a}\left(1+\frac{1}{2}\left(\pi^{*}\left(K_{X}+G_{i}\right)+\pi^{*} L\right)\left(\pi^{*} L\right) F_{i}^{\prime \prime}\right) \\
& +\sum_{i=1}^{m}\left(1+\frac{1}{2}\left(\pi^{*}\left(K_{X}+Z_{i}\right)+\pi^{*} L\right)\left(\pi^{*} L\right) Z_{i}^{\prime \prime}\right) .
\end{aligned}
$$

By the same argument as in the proof of Claim 2.4, we can prove that

$$
\left(\pi^{*}\left(K_{X}+G_{i}\right)+\pi^{*} L\right)\left(\pi^{*} L\right) F_{i}^{\prime \prime} \geq\left(K_{X^{\prime \prime}}+F_{i}^{\prime \prime}+\pi^{*} L\right)\left(\pi^{*} L\right) F_{i}^{\prime \prime}
$$

and

$$
\left(\pi^{*}\left(K_{X}+Z_{i}\right)+\pi^{*} L\right)\left(\pi^{*} L\right) Z_{i}^{\prime \prime} \geq\left(K_{X^{\prime \prime}}+Z_{i}^{\prime \prime}+\pi^{*} L\right)\left(\pi^{*} L\right) Z_{i}^{\prime \prime}
$$


So we obtain that

$$
\begin{aligned}
g(L) \geq & \sum_{i=1}^{a}\left(1+\frac{1}{2}\left(K_{X^{\prime \prime}}+F_{i}^{\prime \prime}+\pi^{*} L\right)\left(\pi^{*} L\right) F_{i}^{\prime \prime}\right) \\
& +\sum_{i=1}^{m}\left(1+\frac{1}{2}\left(K_{X^{\prime \prime}}+Z_{i}^{\prime \prime}+\pi^{*} L\right)\left(\pi^{*} L\right) Z_{i}^{\prime \prime}\right) \\
= & \sum_{i=1}^{a} g\left(\pi^{*} L_{F_{i}^{\prime \prime}}\right)+\sum_{i=1}^{m} g\left(\pi^{*} L_{Z_{i}^{\prime \prime}}\right) .
\end{aligned}
$$

We remark that $g\left(\pi^{*} L_{Z_{i}^{\prime \prime}}\right) \geq 0$ for any $i$ since $\operatorname{dim} Z_{i}^{\prime \prime}=2$.

Case (I). $g(C)=0$. Since $h^{0}\left(\pi^{*} L_{F_{i}^{\prime \prime}}\right) \geq 1$ and $\operatorname{dim} F_{i}^{\prime \prime}=2$, we have $g\left(\pi^{*} L_{F_{i}^{\prime \prime}}\right) \geq$ $q\left(F_{i}^{\prime \prime}\right)$ for any $i$. Because of $q\left(F_{i}^{\prime \prime}\right) \geq q\left(X^{\prime \prime}\right)=q\left(X^{\prime}\right)=q(X)$ for any $i$, we obtain that $g(L) \geq a q(X)$.

Case (II). $g(C) \geq 1$.

Then $\theta=$ id. Since $L$ is ample and $G_{i}$ is a fiber of $f^{\prime}$, there exists a $Z_{i}$ such that $Z_{i} \rightarrow C$ is surjective. We consider the fiber space $Z_{i}^{\prime \prime} \rightarrow C$. By Theorem 2.1 and Theorem 5.5 in [Fk1], $g\left(\pi^{*} L_{Z_{i}^{\prime \prime}}\right) \geq g(C)$ for some $i$. On the other hand, $g\left(\pi^{*} L_{F_{i}^{\prime \prime}}\right) \geq$ $q\left(F_{i}^{\prime \prime}\right)$ because $h^{0}\left(\pi^{*} L_{F_{i}^{\prime \prime}}\right) \geq 1$ and $\operatorname{dim} F_{i}^{\prime \prime}=2$. Hence $g(L) \geq g(C)+a q\left(F_{i}^{\prime \prime}\right)$. Since $g(C)+q\left(F_{i}^{\prime \prime}\right) \geq q\left(X^{\prime \prime}\right)=q\left(X^{\prime}\right)=q(X)$ and $q\left(F_{i}^{\prime \prime}\right)=q\left(F^{\prime}\right)$ for any $i$, we obtain that $g(L) \geq q(X)+(a-1) q\left(F^{\prime}\right)$. (We remark that $a \geq 2$ in this case.)

This completes the proof of this Theorem.

By Theorem 1.6 and Theorem 1.13, we can prove the following Theorem by the same method as that used in the proof of Theorem 2.8 (cf. Theorem 2.7).

Theorem 2.10. Let $(X, L)$ be a polarized 4-fold with $h^{0}(L) \geq 2$. We use Notation 2.6. Then

$$
g(L) \geq g(C)+a g\left(\theta^{*} L_{F^{\prime}}\right)
$$

unless $\Delta(L)=0$ or $(X, L)$ is a scroll over a curve.

In particular, if $a=1$ and $h^{0}(L) \geq 3$, then $g(L) \geq q(X)$ by Theorem 2.1.

Theorem 2.11. Let $(X, L)$ be a polarized 3-fold with $h^{0}(L) \geq 2$. We use Notation 2.6.

If $a=1$, then $g(L) \geq q(X)+\frac{1}{2} G Z L$ unless $\Delta(L)=0$ or $(X, L)$ is a scroll over a curve, where $G$ is a general element of $\Lambda_{M}$.

In particular, $g(L) \geq q(X)+1$ if $Z \neq 0$.

Proof. We remark that the strict transform of $G$ by $\theta$ is $F^{\prime}$. So we have

$$
\begin{aligned}
g(L) & =1+\frac{1}{2}\left(K_{X^{\prime}}+2 \theta^{*} L\right)\left(\theta^{*} L\right)^{2} \\
& =1+\frac{1}{2} \theta^{*}\left(K_{X}+2 L\right)\left(\theta^{*} L\right)^{2} \\
& \geq 1+\frac{1}{2} \theta^{*}\left(K_{X}+2 L\right)\left(\theta^{*} L\right) F^{\prime} \\
& =1+\frac{1}{2}\left(\theta^{*}\left(K_{X}+G\right)+\theta^{*}(L-G)+\theta^{*} L\right)\left(\theta^{*} L\right) F^{\prime} .
\end{aligned}
$$


By using the same argument as in the proof of Claim 2.4, we can prove $\theta^{*}\left(K_{X}+G\right)\left(\theta^{*} L\right) F^{\prime} \geq\left(K_{X^{\prime}}+F^{\prime}\right)\left(\theta^{*} L\right) F^{\prime}$. On the other hand, $\theta^{*}(L-G)\left(\theta^{*} L\right) F^{\prime}=$ $Z G L$. Hence

$$
\begin{aligned}
g(L) & \geq 1+\frac{1}{2}\left(K_{X^{\prime}}+F^{\prime}+\theta^{*} L\right)\left(\theta^{*} L\right) F^{\prime}+\frac{1}{2} Z G L \\
& =g\left(\theta^{*} L_{F^{\prime}}\right)+\frac{1}{2} Z G L .
\end{aligned}
$$

Since $h^{0}\left(\theta^{*} L_{F^{\prime}}\right) \geq 1$ and $\operatorname{dim} F^{\prime}=2$, we obtain that $g\left(\theta^{*} L_{F^{\prime}}\right) \geq q\left(F^{\prime}\right)$.

Therefore

$$
\begin{aligned}
g(L) & \geq q\left(F^{\prime}\right)+\frac{1}{2} Z G L \\
& \geq q(X)+\frac{1}{2} Z G L .
\end{aligned}
$$

If $Z \neq 0$, then $Z \cap G \neq \phi$ since $G+Z$ is connected. Since $L$ is ample and $G$ is a general element of $\Lambda_{M}$, we have $Z G L>0$. Because $g(L)$ is integer, $g(L) \geq$ $q(X)+1$.

Theorem 2.12. Let $(X, L)$ be a polarized 3-fold with $h^{0}(L) \geq 3$. If $g(L)=q(X)$, then $(X, L)$ is one of the following types:

1. $\Delta(L)=0$,

2. $(X, L)$ is a scroll over a curve.

Proof. We use Notation 2.6.

(Step 1). First we assume that $(X, L)$ is not obtained by a finite number of simple blowing up of another polarized 3 -fold.

If $K_{X}+2 L$ is not nef, then $(X, L)$ is the type (1) or (2). So we may assume that $K_{X}+2 L$ is nef.

(1-1) The case in which $g(C) \geq 1$.

We remark that $\theta=$ id in this case. By Theorem 2.8, $q(X)=g(L) \geq q(X)+$ $(a-1) q\left(F^{\prime}\right)$. Since $a \geq 2$, we obtain that $q\left(F^{\prime}\right)=0$. So we have $g(L)=q(X)=$ $g(C)$. But then $\left(f^{\prime}, X, C, L\right)$ is a scroll by Theorem 1.3. This is a contradiction by assumption.

(1-2) The case in which $g(C)=0$.

If $a \geq 2$, then $q(X)=g(L) \geq 2 q(X)$ by Theorem 2.8. Hence $q(X)=0$. Therefore $g(L)=q(X)=0$. By Theorem 1.16, $\Delta(L)=0$ in this case. This is a contradiction by hypothesis.

So we consider the case $a=1$. By Theorem 2.11, $Z=0$, that is, $|L|$ has no fixed component. By the proof of Theorem 2.8, we have $g\left(\theta^{*} L_{F^{\prime}}\right)=q\left(F^{\prime}\right)$. Since $h^{0}\left(\theta^{*} L_{F^{\prime}}\right) \geq 2$, we have $\kappa\left(F^{\prime}\right)=-\infty$. Since $g\left(\theta^{*} L_{F^{\prime}}\right)=q\left(F^{\prime}\right)$, we can prove the following Claim.

Claim 2.13. $\kappa\left(K_{F^{\prime}}+\theta^{*} L_{F^{\prime}}\right)=-\infty$.

Proof. Assume that $\kappa\left(K_{F^{\prime}}+\theta^{*} L_{F^{\prime}}\right) \geq 0$. Then $g\left(\theta^{*} L_{F^{\prime}}\right) \geq 1$.

Since $g\left(\theta^{*} L_{F^{\prime}}\right)=q\left(F^{\prime}\right)$, a $\left(\theta^{*} L_{F^{\prime}}\right)$-minimalization of $\left(F^{\prime}, \theta^{*} L_{F^{\prime}}\right)$ (see Definition 1.17 ) is a scroll over a curve $B$ by Theorem 3.1 in $[\mathrm{Fk} 1]$. Hence there is a surjective morphism $\pi: F^{\prime} \rightarrow B$ such that a general fiber $F_{\pi}$ of $\pi$ is $\mathbb{P}^{1}$. Hence

$$
\left(K_{F^{\prime}}+\theta^{*} L_{F^{\prime}}\right) F_{\pi}=-1 .
$$


But this is a contradiction because $F_{\pi}$ is nef. This completes the proof of this claim.

On the other hand,

$$
\begin{aligned}
K_{F^{\prime}}+\theta^{*} L_{F^{\prime}} & =\left(K_{X^{\prime}}+F^{\prime}+\theta^{*} L\right)_{F^{\prime}} \\
& =\left(\theta^{*}\left(K_{X}+L\right)+E_{\theta}+F^{\prime}\right)_{F^{\prime}},
\end{aligned}
$$

where $E_{\theta}$ is a $\theta$-exceptional effective divisor.

Since $F^{\prime}$ is a general fiber of $f^{\prime}$, we have $h^{0}\left(\left.\left(E_{\theta}+F^{\prime}\right)\right|_{F^{\prime}}\right) \geq 1$.

If $K_{X}+L$ is nef, $m\left(K_{X}+L\right)$ has no base point by the Base Point Free Theorem (see $[\mathrm{KMM}])$ for large $m$. Hence $h^{0}\left(m\left(\theta^{*}\left(K_{X}+L\right)_{F^{\prime}}\right)\right) \geq 1$. Therefore

$$
h^{0}\left(m\left(\left.\left(\theta^{*}\left(K_{X}+L\right)+E_{\theta}+F^{\prime}\right)\right|_{F^{\prime}}\right)\right)>0 .
$$

But this is a contradiction by Claim 2.13 .

Therefore $K_{X}+L$ is not nef. Hence we use Theorem 1.12 and its notation.

By assumption of (Step 1), the case (a) of Theorem 1.12 cannot occur.

If $(X, L)$ is the case (b0), then $g(L)=q(X)=0$. Therefore $\Delta(L)=0$ by Theorem 1.16 and this is a contradiction by hypothesis.

If $(X, L)$ is the case (b1), then $g(L)=q(X)=g(W)$. But by Theorem 1.3, $(X, L)$ is a scroll over $W$. This is a contradiction by hypothesis.

So we consider the case in which $(X, L)$ is the type (b2).

Let $\varphi: X \rightarrow Y$ be its $\mathbb{P}^{1}$-bundle, where $Y$ is a smooth surface.

Claim 2.14. $\kappa(Y)=-\infty$.

Proof. We remark that $Z=0$. We take a general element $G \in|L|$. Then $G$ is irreducible and reduced, and the strict transform of $G$ by $\theta$ is $F^{\prime}$. Since $L$ is ample, $\left.\varphi\right|_{G}: G \rightarrow Y$ is surjective. Hence we obtain $\kappa(Y)=-\infty$ because $\kappa\left(F^{\prime}\right)=-\infty$. This completes the proof of this Claim.

If $q(Y)=0$, then $g(L)=q(X)=q(Y)=0$. Hence $\Delta(L)=0$ by Theorem 1.16. But this is a contradiction by assumption.

If $q(Y) \geq 1$, we take the Albanese map of $Y \alpha: Y \rightarrow B$, where $B$ is a smooth curve. Then $g(L)=q(X)=q(Y)=g(B)$. Hence by Theorem 1.3, $(\alpha \circ \pi, X, B, L)$ is a scroll. But this is a contradiction by assumption.

(Step 2). Next we assume that $(X, L)$ is obtained by a finite number of simple blowing up of another polarized 3 -fold $(Y, A)$ which is not obtained by a finite number of simple blowing up of another polarized 3 -fold.

Then by (Step 1), $(Y, A)$ is one of the following types because $g(L)=g(A)$ and $q(X)=q(Y)$ :

(A) $\Delta(A)=0$,

(B) $(Y, A)$ is a scroll over a curve.

Let $\pi: X \rightarrow Y$ be its birational morphism.

If $(Y, A)$ is the type $(\mathrm{A})$, then $g(A)=0$. Since $g(L)=g(A)$, we have $g(L)=0$ and $\Delta(L)=0$ by Theorem 1.16. So we assume that $(Y, A)$ is the type (B).

Let $h: Y \rightarrow B$ be its $\mathbb{P}^{2}$-bundle. Then $g(A)=g(B)$. We consider the fiber space $(h \circ \pi, X, B)$. Since $g(L)=g(A)$, we have $g(L)=g(B)$. But then $(h \circ \pi, X, B, L)$ is a scroll by Theorem 1.3. But this is a contradiction because $\pi \neq$ id. This completes the proof of Theorem 2.12.

By considering Theorem 2.12, we propose the following conjecture. 
Conjecture 2.15. Let $(X, L)$ be a polarized manifold with $\operatorname{dim} X=n \geq 4$ and $h^{0}(L) \geq n$. If $g(L)=q(X)$, then $(X, L)$ is one of the following types:

1. $\Delta(L)=0$,

2. $(X, L)$ is a scroll over a smooth curve.

\section{REFERENCES}

[B1] L. Bădescu, On ample divisors, Nagoya Math. J. 86 (1982), 155-171. MR 83j:14008

[B2] , On ample divisors II, Proceedings of the "Week of Algebraic Geometry" (Bucharest) 1980 Teubner Texte Math 40 (1981), 12-32. MR 84k:14004

[B3] , The projective plane blown-up at a point as an ample divisor, Atti. Accad. Ligure 38 (1981), 3-7. MR 85e:14055

[B-S] M. C. Beltrametti and A. J. Sommese, The adjunction theory of complex projective varieties, de Gruytev Expositions in Math. 16. MR 96f:14004

[Fj0] T. Fujita, Classification Theories of Polarized Varieties, London Math. Soc. Lecture Note Series 155 (1990). MR 93e:14009

[Fj1]__ On polarized manifolds whose adjoint bundles are not semipositive, Advanced Studies in Pure Math 10 (1985), 167-178. MR 89d:14006

[Fj2] _ Remarks on quasi-polarized varieties, Nagoya Math. J 115 (1989), 105-123. MR 90i: 14045

[Fk1] Y. Fukuma, A lower bound for the sectional genus of quasi-polarized surfaces, Geometriae Dedicata 64 (1997), 229-251. MR 98c:14007

[Fk2] , A lower bound for sectional genus of quasi-polarized manifolds, J. Math. Soc. Japan 49 (1997), 339-362. CMP 98:07

[Fk3] _ A lower bound for sectional genus of quasi-polarized manifolds II, preprint.

[Fk4] - On sectional genus of quasi-polarized manifolds with non-negative Kodaira dimension, Math. Nachr. 180 (1996), 75-84. MR 97f:14005

[I] P. Ionescu, Generalized adjunction and applications, Math. Proc. Cambridge Philos. Soc. 99 (1986), 457-472. MR 87e:14031

[KMM] Y. Kawamata, K. Matsuda, and K. Matsuki, Introduction to the minimal model problem, Advanced Studies in Pure Math 10 (1985), 283-360. MR 89e:14015

[Ma] H. Maeda, On polarized surfaces of sectional genus three, Sci. Papers College Arts Sci. Univ. Tokyo 37 (1987), 103-112. MR 89h:14027

[Ra] C. P. Ramanujam, Remarks on the Kodaira vanishing theorem, J. Indian Math. Soc. 36 (1972), 41-51. MR 48:8502

[S] A. J. Sommese, On the adjunction theoretic structure of projective varieties, Lecture Notes in Math 1194, 175-213. MR 87m:14049

$[\mathrm{S}-\mathrm{V}]$ A. J. Sommese and Van de Ven, On the adjunction mapping, Math. Ann. 278 (1987), 593-603. MR 88j:14011

Department of Mathematics, Faculty of Science, Tokyo Institute of Technology, Oh-OKayama, Meguro-Ku, TOKyo 152, Japan

Current address: Department of Mathematics, College of Education, Naruto University of Education, Takashima, Naruto-cho, Naruto-shi 772-8502, Japan

E-mail address: fukuma@naruto-u.ac.jp 